\title{
Environmentally-induced effects on a bipartite two-level system: geometric phase and entanglement properties
}

\author{
Fernando C. Lombardo ${ }^{*}$ and Paula I. Villar ${ }^{1,2} \dagger$ \\ 1 Departamento de Física Juan José Giambiagi, \\ FCEyN UBA, Facultad de Ciencias Exactas y Naturales, \\ Ciudad Universitaria, Pabellón I, 1428 Buenos Aires, Argentina \\ 2 Computer Applications on Science and Engineering Department, \\ Barcelona Supercomputing Center (BSC), 29, Jordi Girona 08034 Barcelona, Spain
}

(Dated: today)

\begin{abstract}
We calculate the geometric phase of a bipartite two-level system coupled to an external environment. We analyze the reduced density matrix for an arbitrary initial state of the composite system and compute the correction to the unitary geometric phase through a kinematic approach. In all cases considered, we observe a similar structure as a function of the degree of the entanglement of the initial state. Further, we compute the entanglement entropy and concurrence of the bipartite state and analyze if there is any relation among these quantities and the geometric phase acquired during the nonunitary system's evolution. Finally, we discuss the results obtained.

PACS numbers: 03.65.Vf,03.65.Ud,03.67.Pp
\end{abstract}

\section{INTRODUCTION}

It is plausible to imagine a quantum computer as a device made of qubits which interact among themselves in some way. Therefore, a quantum computer can be understood as an interacting quantum many body system. In the last years, several results have been obtained which suggest that entanglement is at the root of the power of quantum computers [1].

A qubit is a quantum two-level system, i.e. a physical system described in terms of a Hilbert space $C^{2}$. This can be considered as a spin- $1 / 2$ particle, or as an atom with two energy levels or even a photon with two possible orthogonal polarizations. Solid state examples which have achieved some experimental success include spins in semiconductors and quantum dots, various designs based on superconductors, vacancy centers in diamond and singles molecule magnets (SMMs), see 2] and references therein.

It is has been shown that one can describe a quantum information processing system in terms of interacting qubits such as SMMs. Quite generally, in doing so, we need to include both environmental nonlocalized modes (like phonons and photons) and discrete localized modes (like defects, nuclear spins, loose spins). The spin-boson model corresponds to a single two-level system interacting with a large reservoir of bosonic field modes, i.e. a spin- $1 / 2$ particle coupled to an environment of harmonic oscillators. The seminal review paper by Legget et al. [3] discusses its dynamics in great detail. The spin-spin model is its analogous but when the spin- $1 / 2$ particle is coupled to an environment of spin- $1 / 2$ particles. The effect of this type of environment on the central spin has also been studied thoroughly by Zurek in Ref.[4].

\footnotetext{
*lombardo@df.uba.ar

†paula@df.uba.ar
}

When coherent superposition states on macroscopic scales are built, they are tipically created at very low temperatures in order to "freeze out" the thermal environment and thus minimize the decoherence effects. This thermal environment can be modelled into a bath of delocalized bosonic modes, implying an interaction among a large spatial region. But lowering the temperature does not affect the influence of localized modes such as nuclear spins or impurities that are intrinsically present in the material [5].

From another point of view, a system can retain the information of its motion when it undergoes a cyclic evolution, in the form of a geometric phase (GP), which was first put forward by Pancharatnam in optics [6] and later studied explicitly by Berry in a general quantal system 7]. Since then, great progress has been achieved in this field. The geometric phase has been extended to the case of non-adiabatic evolutions [8]. As an important evolvement, the application of the geometric phase has been proposed in many fields, such as the geometric quantum computation. Due to its global properties, the geometric phase is propitious to construct fault tolerant quantum gates. In this line of work, many physical systems have been investigated to realize geometric quantum computation, such as NMR (Nuclear Magnetic Resonance), Josephson junction, Ion trap and semiconductor quantum dots. The quantum computation scheme for the geometric phase has been proposed based on the Abelian or non-Abelian geometric phase, in which geometric phase has been shown to be robust against faults in the presence of some kind of external noise due to the geometric nature of Berry phase. It was therefore seen that interactions play an important role for the realization of some specific operations. Consequently, the study of the geometric phase was soon extended to open quantum systems. Following this idea, many authors have analyzed the correction to the geometric phase under the influence of an external environment using different ap- 
proaches $[9-15]$.

In this context, we shall briefly review the way the geometric phase can be computed for a system under the influence of external conditions such as an external bath. In Ref. 9], a quantum kinematic approach was proposed and the geometric phase (GP) for a mixed state under nonunitary evolution has been defined as

$$
\begin{aligned}
\phi_{G}= & \arg \left\{\sum_{k} \sqrt{\varepsilon_{k}(0) \varepsilon_{k}(\tau)}\left\langle\Psi_{k}(0) \mid \Psi_{k}(\tau)\right\rangle \times\right. \\
& \left.e^{-\int_{0}^{\tau} d t\left\langle\Psi_{k}\left|\frac{\partial}{\partial t}\right| \Psi_{k}\right\rangle}\right\},
\end{aligned}
$$

where $\varepsilon_{k}(t)$ are the eigenvalues and $\left|\Psi_{k}\right\rangle$ the eigenstates of the reduced density matrix $\rho_{\mathrm{r}}$ (obtained after tracing over the reservoir degrees of freedom). In the last definition, $\tau$ denotes a time after the total system completes a cyclic evolution when it is isolated from the environment. Taking into account the effect of the environment, the system no longer undergoes a cyclic evolution. However, we shall consider a quasi cyclic path $\mathcal{P}: t \in[0, \tau]$, with $\tau=2 \pi / \Omega$ ( $\Omega$ is the system's characteristic frequency). When the system is open, the original GP, i.e. the one that would have been obtained if the system had been closed $\phi_{G}^{U}$, is modified. This means, in a general case, the phase is $\phi_{G}=\phi_{G}^{U}+\delta \phi_{G}$, where $\delta \phi_{G}$ depends on the kind of environment coupled to the main system 10 12, 16, 17]. If the eigenvalues of the density matrix are degenerate, the expression for the geometric phase takes a slightly different form, as described in [9].

In this paper, we shall study the geometric phase acquired by a bipartite system in the presence of an external environment. We shall consider both the presence of a bosonic and spin environment. We shall choose an arbitrary initial state and see how the geometric phase acquired during the evolution of the composite system is corrected by the presence of an environment. We will show the dependence of the environmentally induced correction to the geometric phase upon the degree of entanglement in the bipartite system. We will also show that, even in the nonunitary evolution, there is no correction to the phase when the composite system is in a maximally entangled state (MES). In this case, we show that the system adopt a total phase $\phi_{G}^{U}=\pi$ which, as it has been shown in previous articles, is of topological nature [18]. Furthermore, we shall also study the entanglement measures of the bipartite system and analyze the relation among them and the geometric phase.

This paper is organized as follows. In Section II, we study the evolution, geometric phase, and entanglement properties of the bipartite system in the presence of a bosonic environment at zero temperature. In Section III, we follow the same procedure for a spin environment leaving its trail in the dynamics of the bipartite system. Finally, we conclude our results in Section IV. Two appendices complete the presentation.

\section{BIPARTITE SPIN-BOSON MODEL}

Oscillator environments correspond to a quasi continuum of delocalized bosonic field modes, with coherence and energy from the central system becoming effectively and irreversibly lost into this extended bosonic environment.

We shall consider a bipartite system, that is to say, two interacting two-level systems, both coupled to an external reservoir.
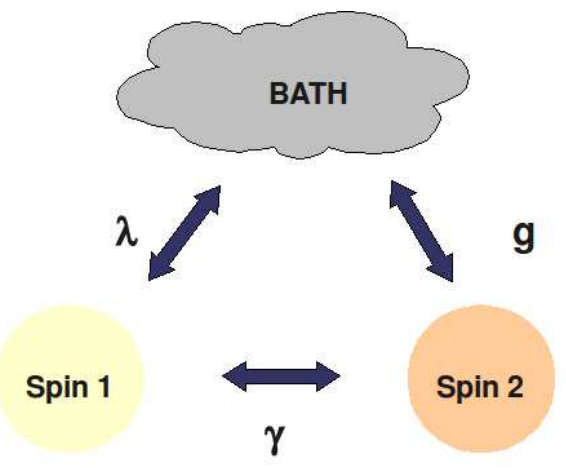

FIG. 1: Diagram of the model we shall consider: two two-level systems coupled to an external reservoir whether it is bosonic or comprised of spins.

In terms of the Hamiltonians, the model can be mathematically described by the Hamiltonian of the free bipartite system $H_{S}$, the Hamiltonian of interaction between the bipartite and the external bath $H_{I}$ and the free Hamiltonian of the external bath $H_{B}$ :

$$
\begin{aligned}
H_{S} & =\frac{\hbar \Omega_{1}}{2} \sigma_{z}^{1}+\frac{\hbar \Omega_{2}}{2} \sigma_{z}^{2}+\gamma \sigma_{z}^{1} \otimes \sigma_{z}^{2} \\
H_{I} & =\sigma_{z}^{1} \otimes \sum_{n=1}^{N} \lambda_{n} q_{n}+\sigma_{z}^{2} \otimes \sum_{n=1}^{N} g_{n} q_{n} \\
H_{B} & =\sum_{n=1}^{N} \hbar \omega_{n} a_{n}^{\dagger} a_{n},
\end{aligned}
$$

where the constants $\lambda_{n}$ and $g_{n}$ couple the system to each oscillator in the environment, and $\gamma$ is the coupling strength between both spin-1/2 particles (as shown in Fig(1). Here we have assumed that each coupling constant of the two level systems with the environment is different being $\lambda_{n}$ for the spin 1 and $g_{n}$ for spin 2 .

In order to compute the geometric phase for the bipartite system it is important to know its dynamics at all times. Therefore, in Appendix $\AA$ we have derivated the reduced density matrix of the bipartite system. It is important to know that this derivation has been done in the weak coupling limit for a general environment defined by a spectral function $J(\omega)$. Following the mentioned derivation, if one consideres the most general case for an initial state of the bipartite system, namely

$$
|\Phi(0)\rangle=\alpha|00\rangle+\beta|01\rangle+\zeta|10\rangle+\delta|11\rangle
$$




$$
\rho_{\mathrm{r}}(t)=\left(\begin{array}{cccc}
|\alpha|^{2} & \alpha \beta^{*} e^{-i\left(2 \gamma+\Omega_{2}\right) t} \Gamma_{2} \Lambda_{12} & \alpha \zeta^{*} e^{-i\left(2 \gamma+\Omega_{1}\right) t} \Gamma_{1} \Lambda_{12} & \alpha \delta^{*} e^{-i\left(\Omega_{1}+\Omega_{2}\right) t} \Gamma_{1} \Gamma_{2} \Gamma_{12}^{2} \\
\beta \alpha^{*} e^{i\left(2 \gamma+\Omega_{2}\right) t} \Gamma_{2} \Lambda_{12}^{*} & |\beta|^{2} & \beta \zeta^{*} e^{-i\left(\Omega_{1}-\Omega_{2}\right) t} \Gamma_{1} \Gamma_{2} \tilde{\Gamma}_{12}^{2} & \beta \delta^{*} e^{-i\left(\Omega_{1}-2 \gamma\right) t} \Gamma_{1} \Lambda_{12}^{*} \\
\zeta \alpha^{*} e^{i\left(2 \gamma+\Omega_{1}\right) t} \Gamma_{1} \Lambda_{12}^{*} & \zeta \beta^{*} e^{i\left(\Omega_{1}-\Omega_{2}\right) t} \Gamma_{1} \Gamma_{2} \tilde{\Gamma}_{12}^{2} & |\zeta|^{2} & \zeta \delta^{*} e^{-i\left(\Omega_{2}-2 \gamma\right) t} \Gamma_{2} \Lambda_{12}^{*} \\
\delta \alpha^{*} e^{i\left(\Omega_{1}+\Omega_{2}\right) t} \Gamma_{1} \Gamma_{2} \Gamma_{12}^{2} & \delta \beta^{*} e^{i\left(\Omega_{1}-2 \gamma\right) t} \Gamma_{1} \Lambda_{12} & \delta \zeta^{*} e^{i\left(\Omega_{2}-2 \gamma\right) t} \Gamma_{2} \Lambda_{12} & |\delta|^{2}
\end{array}\right),
$$

where we have not written explicitly the dependence upon the time of $\Gamma_{i}(t)$ just to simplify notation and we have defined

$$
\tilde{\Gamma}_{12}^{2}=\tilde{\Gamma}_{12}^{2}(t)=e^{+8 \int_{0}^{t} d t_{1} F_{12}\left(t_{1}\right)}
$$

and

$$
\Lambda_{12}=\Lambda_{12}(t)=e^{\mathrm{i} 4 \int_{0}^{t} d t_{1} G_{12}\left(t_{1}\right)}
$$

with $\Gamma_{1}, \Gamma_{2}, F_{i}(t)$ and $G_{i}(t)$ as defined in Appendix A Notice that the coupling constants of the model $\lambda_{n}$ and $g_{n}$ are absorbed in the dimensionless dissipative constants $\gamma_{0_{1}} \sim \lambda^{2}, \gamma_{0_{2}} \sim g^{2}$, and $\gamma_{0_{12}} \sim \lambda g$ respectively, defined in the spectral density of the bath (see Appendix A for details).

In order to have a complete description of the effect of the bath on the bipartite system, we shall consider a quantum ohmic environment at zero temperature and different couplings between both spin-particles and the bath. Consequently, there will be different decoherence factors (see Appendix $\mathrm{A}$ ) and we shall use subindexes 1,2 , or 12 to refer to them. By the definition of the particular initial state, the reduced density matrix and consequently, the dynamics of the open bipartite system is known.

For the particular purpose of this work, we shall define an initial density matrix of the form (by setting the corresponding values of Eq.(5) )

$$
\rho_{\mathrm{r}}(0)=\frac{1-r}{4} I+r|\phi\rangle\langle\phi|,
$$

where $r \epsilon(0,1]$ determines the mixing of the state and $I$ is the unit matrix in the Hilbert space $2 \times 2$. The state $|\phi\rangle$ may be any of the following states,

$$
\begin{aligned}
& |\vartheta\rangle=\sqrt{1-p}|00\rangle+\sqrt{p}|11\rangle \\
& |\mu\rangle=\sqrt{1-p}|01\rangle+\sqrt{p}|10\rangle
\end{aligned}
$$

where $p$ determines the degree of entanglement being $|0\rangle,|1\rangle$ eigenstates of the Pauli operator $\sigma_{z}$. It is easy to note that when $p=1 / 2$, Eqs.(9) and (10) are Bell states and Eq.(8) defines the so-called Werner states which play an important role in quantum information processing. We use Eq. (8) because it includes all possible cases, such as pure or mixed states and maximal or non-maximal entangled states. The first term in Eq. (8) can be regarded as the noise and the mixing coefficient $r$ describes the intensity of noise. Recently the one-to-one correspondence between $r$ of the Werner state and the temperature $\mathrm{T}$ of the one-dimensional Heisenberg two-spin chain with a magnetic field $\mathrm{B}$ along the $\mathrm{z}$-axis, has been established [19].

Finally, if we assume an ohmic environment at zero temperature, the decoherence factors take the following forms,

$$
\begin{aligned}
\Gamma_{1}(t) & =e^{-2 \gamma_{0_{1}} \log \left(1+\Lambda^{2} t^{2}\right)}, \\
\Gamma_{2}(t) & =e^{-2 \gamma_{0_{2}} \log \left(1+\Lambda^{2} t^{2}\right)}, \\
\Gamma_{12}(t) & =e^{-2 \gamma_{0_{12}} \log \left(1+\Lambda^{2} t^{2}\right)},
\end{aligned}
$$

similarly to the spin-boson model for zero temperature [10], where $\Lambda$ is the environmental frequency cutoff.

At this stage, we know the dynamics of the bipartite system for all times. However, we are interested in the geometric phase adquired by the composite system in one quasicyclic evolution $\tau \sim 2 \pi / \Omega$, being $\Omega$ the characteristic frequency of the bipartite system. As we have mentioned in the Introduction, the kinematic approach to the geometric phase can be done by the use of the reduced density matrix. Therefore, we can compute the eigenvalues and eigenvectors of the matrix and compute the geometric phase of this state.

\section{A. Werner state with $|\vartheta\rangle$}

We shall start by assuming an initial density matrix of the form of Eqs. (8) and (9). In such a case, it is possible to write the reduced density matrix for all time $t>0$ as 


$$
\rho_{\mathrm{r}_{\mathrm{A}}}(t)=\left(\begin{array}{cccc}
\frac{(1-r)}{4}+r(1-p) & 0 & 0 & r \sqrt{p(1-p)} e^{-\mathrm{i}\left(\Omega_{1}+\Omega_{2}\right) \mathrm{t}} \Gamma_{1}(t) \Gamma_{2}(t) \Gamma_{12}^{2}(t) \\
0 & \frac{(1-r)}{4} & 0 & 0 \\
0 & 0 & \frac{(1-r)}{4} & 0 \\
r \sqrt{p(1-p)} e^{\mathrm{i}\left(\Omega_{1}+\Omega_{2}\right) \mathrm{t}} \Gamma_{1}(t) \Gamma_{2}(t) \Gamma_{12}^{2}(t) & 0 & 0 & \frac{(1-r)}{4}+r p
\end{array}\right) .
$$

In this case, the eigenvalues of Eq. (14) are

$$
\begin{aligned}
\varepsilon_{+}(t) & =\frac{1}{4}\left(1+r+2 r \sqrt{1+4 p(1-p)\left(\Gamma_{12}^{4} \Gamma_{1}^{2} \Gamma_{2}^{2}-1\right)}\right), \\
\varepsilon_{-}(t) & =\frac{1}{4}\left(1+r-2 r \sqrt{1+4 p(1-p)\left(\Gamma_{12}^{4} \Gamma_{1}^{2} \Gamma_{2}^{2}-1\right)}\right), \\
\varepsilon_{1} & =\frac{1-r}{4} \\
\varepsilon_{2} & =\frac{1-r}{4},
\end{aligned}
$$

with the corresponding eigenvectors,

$$
\begin{aligned}
\left|\Psi_{+}\right\rangle & =\frac{r \sqrt{p(1-p)} e^{-\mathrm{i}\left(\Omega_{1}+\Omega_{2}\right) \mathrm{t}} \Gamma_{12}^{2} \Gamma_{1} \Gamma_{2}}{\sqrt{\left(\varepsilon_{+}-(1-p)\right)^{2}+p(1-p) r^{2} \Gamma_{1}^{2} \Gamma_{2}^{2} \Gamma_{12}^{4}}}|00\rangle \\
& +\frac{\left(\varepsilon_{+}-(1-p)\right)}{\sqrt{\left(\varepsilon_{+}-(1-p)\right)^{2}+p(1-p) r^{2} \Gamma_{1}^{2} \Gamma_{2}^{2} \Gamma_{12}^{4}}}|11\rangle \\
\left|\Psi_{-}\right\rangle & =\frac{r \sqrt{p(1-p)} e^{-\mathrm{i}\left(\Omega_{1}+\Omega_{2}\right) \mathrm{t}} \Gamma_{12}^{2} \Gamma_{1} \Gamma_{2}}{\sqrt{\left(\varepsilon_{-}-(1-p)\right)^{2}+p(1-p) r^{2} \Gamma_{1}^{2} \Gamma_{2}^{2} \Gamma_{12}^{4}}}|00\rangle \\
& +\frac{\left(\varepsilon_{-}-(1-p)\right)}{\sqrt{\left(\varepsilon_{-}-(1-p)\right)^{2}+p(1-p) r^{2} \Gamma_{1}^{2} \Gamma_{2}^{2} \Gamma_{12}^{4}}}|11\rangle \\
\left|\Psi_{1}\right\rangle & =|01\rangle \\
\left|\Psi_{2}\right\rangle & =|10\rangle .
\end{aligned}
$$

We see that, with this particular choice of the initial state, we are left to work in the space spanned by $|00\rangle$ and $|11\rangle$, since $\left|\Psi_{1,2}\right\rangle$ are time independent and hence will not contribute to the geometric phase. Using Eq.(11), formally the geometric phase can be computed as

$$
\begin{aligned}
\phi_{G}= & \arg \left\{\sqrt{\varepsilon_{+}(0) \varepsilon_{+}(\tau)}\left\langle\Psi_{+}(0) \mid \Psi_{+}(\tau)\right\rangle\right. \\
& e^{i \Omega \int_{0}^{\tau} \cos ^{2}\left(\theta_{+}\left(t_{1}\right)\right) d t_{1}} \\
+ & \sqrt{\varepsilon_{-}(0) \varepsilon_{-}(\tau)}\left\langle\Psi_{-}(0) \mid \Psi_{-}(\tau)\right\rangle \\
& \left.e^{i \Omega \int_{0}^{\tau} \cos ^{2}\left(\theta_{-}\left(t_{1}\right)\right) d t_{1}}\right\},
\end{aligned}
$$

by defining

$$
\cos \left(\theta_{ \pm}(t)\right)=\frac{r \sqrt{p(1-p)} \Gamma_{1} \Gamma_{2} \Gamma_{12}^{2}}{\left(\sqrt{\left(\varepsilon_{ \pm}-(1-p)\right)^{2}+p(1-p) \Gamma_{12}^{4} \Gamma_{1}^{2} \Gamma_{2}^{2}}\right)},
$$

in analogy to what has been done for a single two-level system in $\mathrm{SU}(2)$ [9, 10]. In this case, one can use $\tau=2 \pi / \Omega$, with $\Omega=\left(\Omega_{1}+\Omega_{2}\right)$.

In order to compute the geometric phase and obtain concrete results, we shall choose $r=1$. In this case it is easy to see that Eq.(16) simplifies since $\varepsilon_{-}(0)=0$ and the contribution of this eigenenergy and its associated eigenvector to the geometric phase is null. Hence, the the geometric phase becomes

$$
\begin{aligned}
\phi_{G}= & \left(\Omega_{1}+\Omega_{2}\right) \times \\
& \int_{0}^{\tau} d t \frac{(1-p) p \Gamma_{12}^{4} \Gamma_{1}^{2} \Gamma_{2}^{2}}{(1-p) p \Gamma_{12}^{4} \Gamma_{1}^{2} \Gamma_{2}^{2}+\left[\varepsilon_{+}-(1-p)\right]^{2}} . \\
= & \left(\Omega_{1}+\Omega_{2}\right) \int_{0}^{\tau} \cos ^{2}\left(\theta_{+}(t)\right) .
\end{aligned}
$$

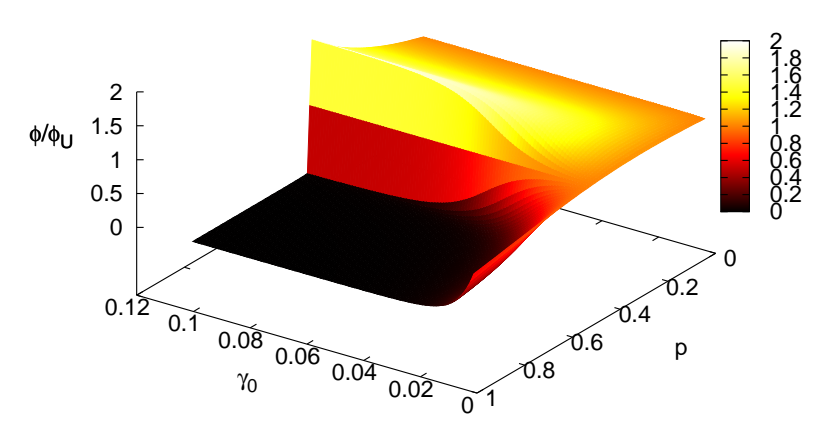

FIG. 2: Deviation from the unitary geometric phase for the bipartite system coupled to an ohmic bosonic environment as a function of the initially entanglement of the state $p$ and the coupling constant $\gamma_{0}$. The frequency cutoff is $\Lambda / \Omega=100$.

It is important to note that if the bipartite system is isolated, then $\Gamma_{i}(t)=1$, and the geometric phase becomes

$$
\phi_{G}=\left(\Omega_{1}+\Omega_{2}\right) \int_{0}^{\tau} d t(1-p)=2 \pi(1-p),
$$

which, of course, is the unitary geometric phase $\phi_{G}^{U}$. We can interpret this result by analogy to a single spin-boson model. In Ref. [10], we have started by a pure initial state on the Bloch Sphere, characterized by $\theta_{0}$, i.e. $|\Phi(0)\rangle=$ $\cos \left(\theta_{0} / 2\right)|0\rangle+\sin \left(\theta_{0} / 2\right)|1\rangle$. Then, in our present case, it is 
enough to define $\sqrt{1-p}=\cos \left(\theta_{0} / 2\right)$. Then the unitary geometric phase becomes $\phi_{G}^{U}=2 \pi \cos ^{2}\left(\theta_{0} / 2\right)=\pi(1+$ $\left.\cos \theta_{0}\right)$, which agrees with the geometric phase acquired by a spin in $\mathrm{SU}(2)$. In other words, as the subspace spanned by $\{|01\rangle,|10\rangle\}$ is a decoherence free space, it is not affected by the presence of an environment. Since $\left[H_{S}, H_{I}\right]=0$, in this case, it does not evolve in time (see Eq.(A1)). Then, as long as the whole system is prepared in a separable state, the geometric phase of the composite is exactly that of the evolution happening in the Hilbert Space spanned by $\{|00\rangle,|11\rangle\}$, similarly to that of $2 \times 2$.

It is also important to note that in the case of having a maximally entangled state is $\phi_{G}=\pi$. Physically, this phase depends on the parity of the number of times the state crosses the space orthogonal to the initial state in the representation of a SO3 sphere. It is already known that for a qubit the total phase gained is $\pi$ (or $n \pi$ ) and it is due to a combination of the dynamical phase and the geometric phase. For a two-level bipartite system with an arbitrary degree of entanglement a third possible type of global phase can be identified (in addition to the dynamical and geometric phase already known): a topological phase, which is a consequence of the geometry of the entangled two-level system. This phase has been studied for maximally entangled states (MES) and it is at the origin of singularities appearing in the phase of MES during a cyclic evolution. In [18], it is studied the phase dynamics of entangled qubits under unitary cyclic evolutions. Therein, it is shown that, after a cyclic evolution, the combination of the different phases always leads to a global phase of an entire multiple of $\pi$. This result, already known and verified experimentally for a single qubit is recovered here for an entangled qubit with maximal degree of entanglement in the presence of an environment.

For a bipartite state, we can not longer use the Bloch sphere to seek a geometric representation of that state. In [18] it has been shown that a geometric representantion of a bipartite state can be obtained by using a Bloch ball and a SO3 sphere. Therefore, the total phase gained by a state is a combination of not only the dynamical and geometrical phase, but also the topological phase. Similarly to one qubit states, MES also gain a total phase of $\pi$ (or $n \pi$ ) under a cyclic evolution. However, this phase is of topological origin. In this context, we can explain our results. We are looking to corrections to the unitary phase. However, in the case of a MES there is no correction to the unitary phase. How can this be? We strongly believe that it is due to the nature of the unitary phase. As we have explained above, it is of topological origin. Then, it can not be disturbed or modified by the dynamic of the environment. For all other degree of entanglement, we do obtain a correction displaying the type of behaviours stated in [18], with the interesting additive factor that we are considering the presence of an enviroment.

In Fig 2, we show the behavior of the geometric phase for the open composite system, as a function of $p$ and the coupling constant $\gamma_{0}$, when the bipartite system is cou- pled to a bosonic ohmic environment by the same dimensionless coupling, i.e. $\gamma_{0_{1}}=\gamma_{0_{2}}=\gamma_{0_{12}} \equiv \gamma_{0}$. Therein, we can note that for very small values of the coupling constant, the geometric phase is that of the unitary system, meaning by the latter that there is no interaction of the bipartite with an environment (closed system). When the presence of the environment is relevant enough to influence the dynamics of the bipartite system, the correction to the unitary geometric phase $\delta \phi_{G}$ also depends of the degree of entanglement $p$, being zero when the initial state is maximally entangled $(p=1 / 2)$. This result has some peculiar characteristics similar to the ones observed in [20]. There, authors studied the isolated system and obtained the geometric phase by tracing over one spin as suggested in 21]. Even though we are considering an external environment, we can note the symmetry in $p$ of our result derived from the type of coupling, and also the jump at the crossover point $p=1 / 2\left(\theta_{0}=\pi / 2\right)$, when the initial state falls from the upper to the lower semi-sphere in the Bloch representation.

In order to analitically analyze the correction to the unitary geometric phase we can perform a series expansion in powers of the coupling with the environment $\gamma_{0}$ (assuming a solely coupling between the bipartite system and the environment, i.e. $\left.\gamma_{0_{i}}=\gamma_{0}\right)$ )

$$
\begin{aligned}
\phi_{G} \approx & 2 \pi(1-p)+32 \gamma_{0} p \frac{\Omega}{\Lambda}\left(1-3 p+2 p^{2}\right) \\
& {\left[\arctan \left(2 \pi \frac{\Lambda}{\Omega}\right)\right.} \\
+ & \left.\pi \frac{\Lambda}{\Omega}\left(-2+\log \left(1+4 \pi^{2} \frac{\Lambda^{2}}{\Omega^{2}}\right)\right)\right],
\end{aligned}
$$

where $\Omega=\Omega_{1}+\Omega_{2}$.

Assuming that $\Lambda / \Omega \gg 1$, the correction $\delta \phi_{G}$ to the geometric phase can be well approximated by

$$
\delta \phi_{G} \approx 64 \pi \gamma_{0} p\left(1-3 p+2 p^{2}\right)\left(\log \left(\frac{2 \pi \Lambda}{\Omega}\right)-1\right),
$$

which is essentially the same correction found in [10] for a single two-level particle.

In Fig 3 we show the geometric phase from Eq.(18) and the geometric phase obtained by the use of Eq.(19). The above expressions show that the phase of the composite system depends on several parameters: the coupling constant $\gamma_{0}$, the frequency cutoff $\Lambda$ (i.e. the "size" of the environment) and the degree of entanglement of the initial state $p$. In Fig, 3 , we can observe that the correction grows with $p$ up to $p=1 / 2$. For values of $\gamma_{0} p \ll 1$ the perturbative expression for the geometric phase becomes a good approximation.

We can think of a realistic model where our central system is coupled to an external environment composed of phonons or photons. In such case, we should consider an non-ohmic spectral density for such an environment, particularly one that goes as $J(\omega) \sim \omega^{3}$, usually 


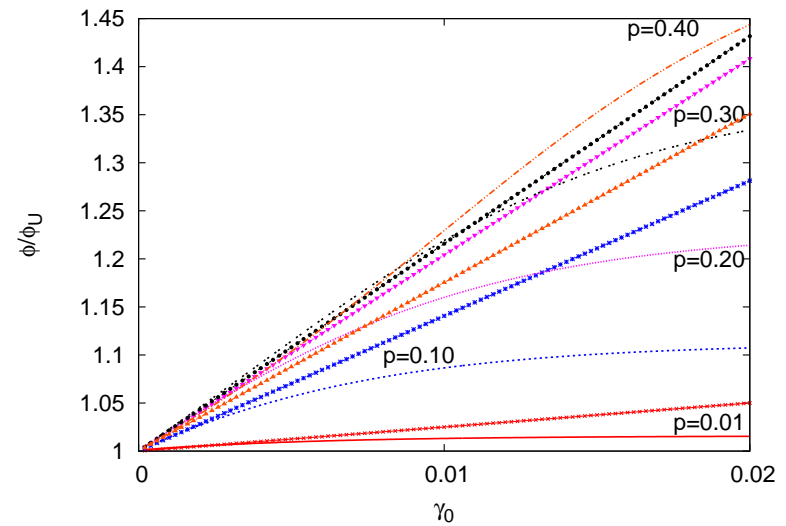

FIG. 3: (colors online) Deviation from the unitary geometric phase for the bipartite system coupled to an ohmic bosonic environment as a function of the initially entanglement of the state $p$ and the coupling constant $\gamma_{0}$. Exact results are plotted with lines and perturbative ones with lines and dots. Equal colors indicate equal values of $p . \Lambda / \Omega=100$.

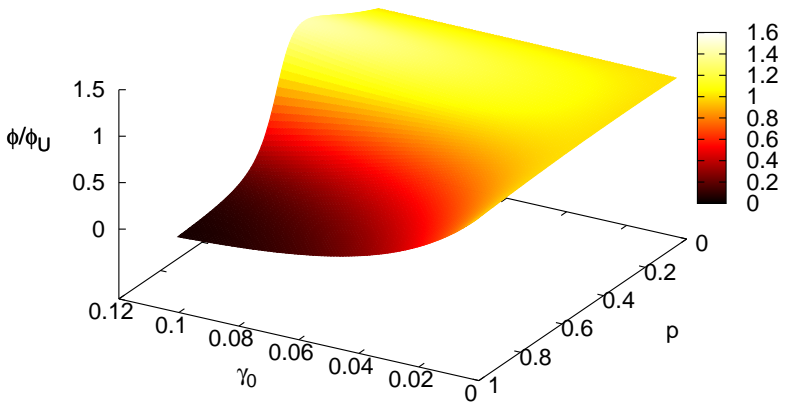

FIG. 4: (colors online) Deviation from the unitary geometric phase for the bipartite system coupled to an supraohmic bosonic environment as a function of the initially entanglement of the state $p$ and the coupling constant $\gamma_{0} . \Lambda / \Omega=100$.

called supraohmic environment [3]. The decoherence factor takes a subtly different form, being decoherence even less effective at zero temperature,

$$
\Gamma^{\text {supra }}(t)=e^{-4 \gamma_{0} \frac{\Lambda^{4} t^{4}}{\left(1+\Lambda^{2} t^{2}\right)^{2}}} .
$$

For times $\Lambda t>1$, this factor becomes $\Gamma^{\text {supra }}(t) \sim e^{-4 \gamma_{0}}$. Since $\gamma_{0}<1$, decoherence is not as effective as in previ-

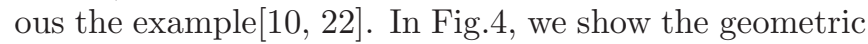
phase for the open bipartite system when it is coupled to a bosonic supraohmic environment by the same coupling constant $\gamma_{0}$. In this case, we see that the effect of the environment over the geometric phase is less relevant since the correction to the unitary geometric phase is smaller than the previous case for the same set of parameters.
A series expansion in powers of the coupling constant $\gamma_{0}$, gives

$$
\begin{aligned}
\phi_{G} \approx & 2 \pi(1-p)+8 \gamma_{0} p \frac{\Omega}{\Lambda}\left(1-3 p+2 p^{2}\right) \\
& {\left[\pi \Lambda\left(\frac{4}{\Omega}+\frac{2 \Omega}{\Omega^{2}+4 \pi^{2} \Lambda^{2}}\right)-3 \arctan \left(2 \pi \frac{\Lambda}{\Omega}\right)\right], }
\end{aligned}
$$

where the correction to the phase can also approximated for $\Lambda / \Omega \gg 1$ as

$$
\delta \phi_{G} \approx 32 \pi \gamma_{0} p\left(1-3 p+2 p^{2}\right)
$$

showing it is smaller than the correction induced in the ohmic case Eq. (20) by a $\log (\Lambda / \Omega)$ factor [10].

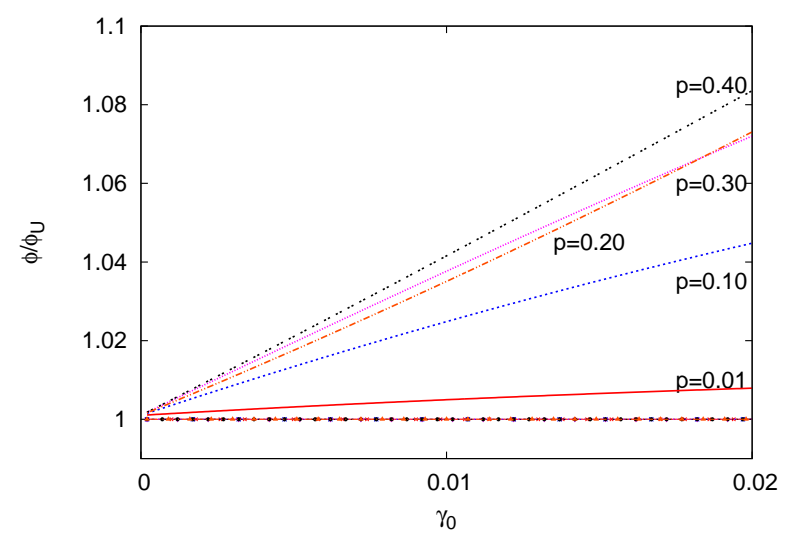

FIG. 5: (colors online) Deviation from the unitary geometric phase for the bipartite system coupled to a nonohmic bosonic environment as a function of the initially entanglement of the state $p$ and the coupling constant $\gamma_{0}$. Exact results are plotted with lines and perturbative ones with lines and dots. Equal colors indicate equal values of $p . \Lambda / \Omega=100$.

In Fig 5 we present the deviation from the unitary geometric phase for the bipartite system coupled to a supraohmic bosonic environment as a function of the degree of entanglement of the initial state $p$ and the coupling constant $\gamma_{0}$. The supraohmic environment is less effective in inducing decoherence on the system and consequently, affects less the system's dynamics, but it also produces a phase correction on the bipartite state.

The correction to the unitary geometric phase naturally depends on the size of the environment, set by $\Lambda$. If we consider bigger or smaller ones, the result will be qualitatively the same [10, 22]. Typically, decoherence from the ohmic bath is larger, limiting the possibility of measuring the correction to the phase using interferometry. 


\section{Linear Entropy and Concurrence}

Entanglement is a quantum-mechanical feature which does not exist in the classical world. It carries non-local correlations between the different parts in such a way that can not be described classically. Bipartite entanglement of pure states is conceptually well understood. A useful measure of many-body entanglement when the total system is in a pure state is the Von Neumann entanglement entropy $S l(t)$, which provides a measure of the bipartite entanglement present in pure states. To be precise, the entanglement entropy measures the optimal rate at which it is possible to distill Bell pairs by local operations in the limit of having an infinite number of copies of the bipartite system.



FIG. 6: (colors online) Concurrence and Linear Entropy as a function of time for different values of the parameter $p$ when the bipartite system is coupled to a bosonic environment with coupling constant $\gamma_{0}=0.002$. Parameters used: $\Lambda=100$, $p=0.01$ solid line, $p=0.20$ dashed lined and $p=0.5$ dotdashed line. The concurrence for a supraohmic environment (with dots) is also indicated for $p=0.01$ and $p=0.5$.

The von Neumann entanglement entropy (or linear entropy) is obtained by focusing on bipartite systems where space can be divided into two regions: the one corresponding to the bipartite system and the one of the environment. After tracing over the degrees of freedom of the environment, we obtain the reduced density matrix of the system $\rho_{\mathrm{r}}$. The von Neumann entanglement entropy is defined as,

$$
S l=-\operatorname{Tr}\left[\rho_{\mathrm{r}} \log _{2}\left(\rho_{\mathrm{r}}\right)\right]
$$

In terms of the eigenvalues of the reduced density matrix of the bipartite system, the entanglement entropy $S l$ reads,

$$
S l(t)=-\varepsilon_{+}(t) \log _{2}\left(\varepsilon_{+}(t)\right)-\varepsilon_{-}(t) \log _{2}\left(\varepsilon_{-}(t)\right) .
$$

Quantum decoherence implies a rapid reduction of the off-diagonal terms of the bipartite reduced density matrix which results in the case of maximal entanglement in $\varepsilon_{ \pm}(t) \rightarrow 1 / 2$ and $S l(t) \rightarrow 1$.

The quantity for measuring the entanglement between the different parts of the composite system is the concurrence [23]. The concurrence for the evolution of this state can be computed as $\mathcal{C}\left(\rho_{c_{\mathrm{r}}}\right)=\max \left(0, \sqrt{\lambda_{1}}-\sqrt{\lambda_{2}}-\right.$ $\left.\sqrt{\lambda_{3}}-\sqrt{\lambda_{4}}\right)$, where $\lambda_{1}, \lambda_{2}, \lambda_{3}$ and $\lambda_{4}$ are the eigenvalues of $\rho_{c_{\mathrm{r}}}=\rho_{\mathrm{r}}^{*}\left(\sigma_{y}^{1} \otimes \sigma_{y}^{2}\right) \rho_{r}\left(\sigma_{y}^{1} \otimes \sigma_{y}^{2}\right)$.

In the case of the initial condition considered in the last Section, the concurrence is

$$
\mathcal{C}_{A}=2 \Gamma^{4}(t) \sqrt{p(1-p)}
$$

In the closed system case, the concurrence is $\mathcal{C}_{A}=1$ when considering a $\operatorname{MES}(p=1 / 2)$, and $\mathcal{C}_{A}=0$ for product sates $(p=0)$. In the open system case, the concurrence depends on the decoherence factor $\Gamma(t)$ as can be seen from Eq.(26), being smaller in the supraohmic case.

In Figs 6 and 7 we show the linear entropy (Eq. 25) and concurrence (Eq.(26) ) when the bipartite system is coupled to the bosonic environment by a solely coupling constant $\gamma_{0}$. Therein, we can see that when the value of $\gamma_{0}$ is small, then the ohmic and supraohmic environments induce the same behaviour on the entanglement of the system state. However, as $\gamma_{0}$ increases, the ohmic environment destroys whatever degree of entanglement might be while the supraohmic is not effective in such a task.

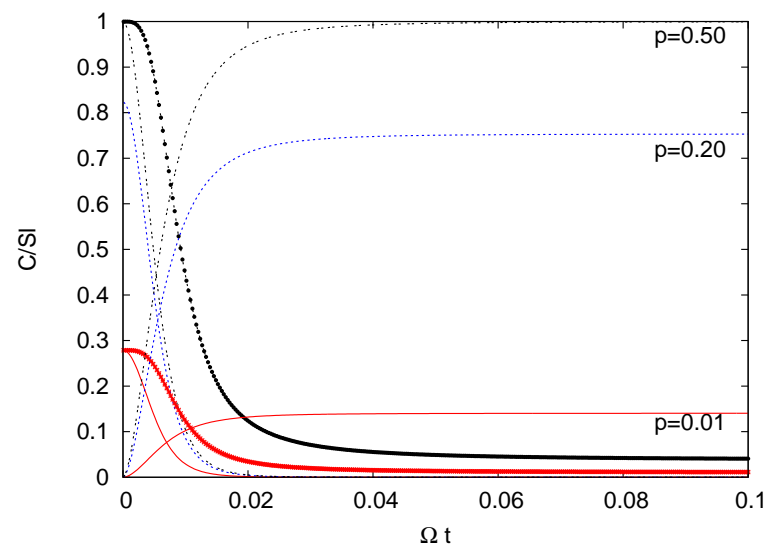

FIG. 7: (colors online) Concurrence and Linear Entropy as a function of time for different values of the parameter $p$ when the bipartite system is coupled to a bosonic environment with coupling constant $\gamma_{0}=0.1$. Parameters used: $\Lambda=100, p=$ 0.01 solid line, $p=0.20$ dashed lined and $p=0.5$ dot-dashed line. The concurrence for a supraohmic environment is also indicated (with dots) for $p=0.01$ and $p=0.5$.

It is worth mentioning that both quantities, i.e the geometric phase and concurrence, are modified by the presence of the environment to a greeter or lesser extend. We can see that for a strong environment $\left(\gamma_{0}=0.1\right)$, and entangled states between $0<p<1 / 2$ and $1 / 2<p<1$, the correction to the geometric phase is important. However, 
for all values of $p$ the concurrence decays at very short times in the case of the ohmic environment. In the case of the supraohmic environment, the situation is much similar to a quasi-isolated system, and the concurrence is not much affected by the noise and dissipation introduced by the external bath.

Finally, it has been stated that there was some kind of correlation among the geometric phase of the individual spins and the concurrence for an entangled state of two spin-1/2 particles [24]. However, we have shown that there is no such relations in the general case (when the coupling to an external environment is considered). Only in the isolated (or unitary) case, it is possible to explicitly show that

$$
\frac{\phi_{G}^{U}}{\mathcal{C}_{A}}=\pi \sqrt{\frac{1-p}{p}}
$$

which is constant for a fixed value of $\mathrm{p}$. In the case of a MES with $p=1 / 2$, the above relation reads $\Phi_{G}^{U}=\pi \mathcal{C}_{A}$ 24].

\section{B. Werner state with $|\mu\rangle$}

Another possibility is to begin with an initial bipartite state of the form $|\mu\rangle$ in Eq.(8). In this case, the reduced density matrix has a slightly simpler expression such as,

$$
\rho_{\mathrm{r}_{\mathrm{B}}}(t)=\left(\begin{array}{cccc}
\frac{(1-r)}{4} & 0 & 0 & 0 \\
0 & \frac{(1-r)}{4}+r(1-p) & r \sqrt{p(1-p)} e^{-i\left(\Omega_{1}-\Omega_{2}\right) t} \Gamma_{1} \Gamma_{2} \tilde{\Gamma}_{12}^{2} & 0 \\
0 & r \sqrt{p(1-p)} e^{i\left(\Omega_{1}-\Omega_{2}\right) t} \Gamma_{1} \Gamma_{2} \tilde{\Gamma}_{12}^{2} & \frac{(1-r)}{4}+r p & 0 \\
0 & 0 & 0 & \frac{(1-r)}{4}
\end{array}\right)
$$

As before, we need to compute the eigenvalues and eigenvectors of the reduced density matrix in order to know the geometric phase of the system. For simplicity, we shall choose again $r=1$. In that case, the eigenvalues will be very similar to those calculated before, with $\varepsilon_{1}=$ $\varepsilon_{2}=0$ and

$$
\begin{aligned}
& \varepsilon_{+}(t)=\frac{1}{2}\left(1+\sqrt{1+4 p(1-p)\left(\tilde{\Gamma}_{12}^{4} \Gamma_{1}^{2} \Gamma_{2}^{2}-1\right)}\right), \\
& \varepsilon_{-}(t)=\frac{1}{2}\left(1-\sqrt{1+4 p(1-p)\left(\tilde{\Gamma}_{12}^{4} \Gamma_{1}^{2} \Gamma_{2}^{2}-1\right)}\right),
\end{aligned}
$$

with the corresponding eigenvectors that can be easily computed as before.

Again, we can see that $\varepsilon_{-}(t=0)=0$. Then, the only contribution to the geometric phase comes from the eigenvalue $\varepsilon_{+}$and its associated eigenvector $\left|\Psi_{+}\right\rangle$. Similarly to the above procedure, the geometric phase is,

$$
\begin{aligned}
\phi_{G}= & \left(\Omega_{1}-\Omega_{2}\right) \times \\
& \int_{0}^{\tau} d t \frac{p(1-p) \Gamma_{1}^{2} \Gamma_{2}^{2} \tilde{\Gamma}_{12}^{4}}{p(1-p) \Gamma_{1}^{2} \Gamma_{2}^{2} \tilde{\Gamma}_{12}^{4}+\left[\varepsilon_{+}-(1-p)\right]^{2}} .
\end{aligned}
$$

From this equation, it is possible to show that there is no correction to the unitary phase in the case of starting whit a MES. Therefore, when $p=1 / 2$ we get $\phi_{G}=\pi$.

As expected, we reobtain the unitary result for the geometric phase if the system is isolated, i.e. $\gamma_{0_{i}}=0$, and consequently $\Gamma_{i}=1$. Furthermore, if we assume that the coupling between each spin and the environment is the same, i.e. $\gamma_{01}=\gamma_{02}=\gamma_{012} \equiv \gamma_{0}$, we see that surprisingly there is no correction to the unitary geometric phase, since

$$
\phi_{G}=\phi_{G}^{U}=2 \pi(1-p)
$$

being at this time $\tau=2 \pi / \Omega$ with $\Omega=\left(\Omega_{1}-\Omega_{2}\right)$.

We can state that in this case the geometric phase is robust against the action of the external environment. This is easily understood since, as we have explained before, the subspace spanned by $|01\rangle,|10\rangle$ is decoherence-free. Solely in the case in which all the coupling strengths are different, one to each other, the total geometric phase accounts for environmentally induced corrections.

Incidentally, if we compute the concurrence for this initial bipartite system state, we see that it is no longer a function of the decoherence factor (when $\gamma_{0 i} \equiv \gamma_{0}$ ),

$$
\begin{aligned}
\mathcal{C}_{B} & =\sqrt{1-p+2 \sqrt{p(1-p)^{3}}} \\
& -\sqrt{1-p-2 \sqrt{p(1-p)^{3}}} .
\end{aligned}
$$

In this case the concurrence $\mathcal{C}_{B}$ does not depends on the decoherence factor and, as in the usual case, we have $\mathcal{C}_{B}=1$ for $p=1 / 2$ and $\mathcal{C}_{B}=0$ for $p=0$. 


\section{BIPARTITE SPIN-SPIN MODEL}

Spin environments are typically the appropriate model in the low temperature regime. In particular, experiments devoted to the studies of macroscopic quantum coherence and decoherence require temperatures close to absolute zero in order to operate. Experimental evidence shows that decoherence is mainly dominated by interactions with localized modes in this setting, such as paramagnetic spins, electronic impurities, defects and nuclear spins. Each of the localized modes is described by a finite-dimensional Hilbert space with a finite energy cutoff. There are numerous examples that show how spin environments influence the dynamics of a central system by becoming a source of decoherence.

In this framework, we examine the decoherence induced by disordered interacting spin baths at finite temperature. Our choice of the bath is the most simple case for an Ising chain so as to facilitate an analytical study of the decoherence and the geometric phase for the model. We shall consider the bipartite two-level system coupled to an external spin environment, modelled by the following Hamiltonians:

$$
\begin{aligned}
& H_{S}=\frac{\hbar}{2}\left(\Omega_{1} \sigma_{z}^{1}+\Omega_{2} \sigma_{z}^{2}\right)+\gamma \sigma_{z}^{1} \otimes \sigma_{z}^{2} \\
& H_{I}=\sigma_{z}^{1} \otimes \sum_{i=1}^{N} \varepsilon_{i} \sigma_{z i}+\sigma_{z}^{2} \otimes \sum_{i=1}^{N} \lambda_{i} \sigma_{z i} \\
& H_{B}=\sum_{i=1}^{N} h_{i} \sigma_{x i} .
\end{aligned}
$$

We have included the free Hamiltonian $H_{B}$ of the environment, where $h_{i}$ denotes the tunneling matrix element for the ith-environmental spin. This free Hamiltonian lends intrinsic dynamics to the environment, in contrast with the more simplified spin-environment models.

As we have done with the bosonic environment, it is imperious to know the dynamics of the system in order to study the geometric phase from the kinematic point of view (Eq.(1)). To that end, we have derived the reduced density matrix of the bipartite system in Appendix B. Herein, we shall consider two different initial states as before (Eqs. (9) and (10)).

\section{A. Werner state con $|\vartheta\rangle$}

Herein, we shall write $|\vartheta\rangle$ in Eq.(8). For this initial state, the reduced density matrix has a much simpler expression than the general one,

$$
\rho_{\mathrm{r}_{\mathrm{A}}}(t)=\left(\begin{array}{c}
\frac{(1-r)}{4}+r(1-p) \\
0 \\
0 \\
r \sqrt{p(1-p)} e^{i\left(\Omega_{2}+\Omega_{1}\right) t} Q^{*}(t)
\end{array}\right.
$$

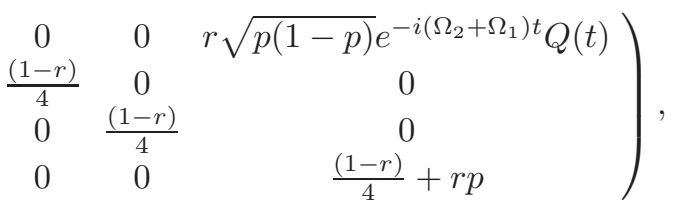

where the decoherence factor $Q(t)$ is defined as,

$$
\begin{aligned}
Q(t) & =\prod_{i=1}^{N}\left\{1-\left(\frac{2\left(\varepsilon_{i}+\lambda_{i}\right)^{2}}{h_{i}^{2}+\left(\varepsilon_{i}+\lambda_{i}\right)^{2}}\right)\right. \\
& \left.\times \sin ^{2}\left(t \sqrt{h_{i}^{2}+\left(\varepsilon_{i}+\lambda_{i}\right)^{2}}\right)\right\}
\end{aligned}
$$

and has been derived in the Appendix $B$.

In order to compute the geometric phase, we need to know the eigenvalues of the reduced density matrix. By setting $r=1$, these are

$$
\begin{aligned}
\epsilon_{1,2} & =0 \\
\epsilon_{ \pm} & =\frac{1}{2}\left[1 \pm \sqrt{1+4(1-p) p\left(Q(t)^{2}-1\right)}\right]
\end{aligned}
$$

and the corresponding eigenvectors

$$
\begin{aligned}
& \left|v_{1}\right\rangle=|01\rangle \\
& \left|v_{2}\right\rangle=|10\rangle
\end{aligned}
$$

and

$$
\begin{aligned}
\left|v_{+}\right\rangle & =\frac{\sqrt{p(1-p)}|Q(t)| e^{-i\left(\Omega_{1}+\Omega_{2}\right) t}}{\sqrt{p(1-p)|Q(t)|^{2}+\left[\epsilon_{+}-(1-p)\right]^{2}}}|00\rangle \\
& +\frac{\left[\epsilon_{+}-(1-p)\right]}{\sqrt{p(1-p)|Q(t)|^{2}+\left[\epsilon_{+}-(1-p)\right]^{2}}}|11\rangle \\
\left|v_{-}\right\rangle & =\frac{\sqrt{p(1-p)}|Q(t)| e^{-i\left(\Omega_{1}+\Omega_{2}\right) t}}{\sqrt{p(1-p)|Q(t)|^{2}+\left[\epsilon_{-}-(1-p)\right]^{2}}}|00\rangle \\
& +\frac{\left[\epsilon_{-}-(1-p)\right]}{\sqrt{p(1-p)|Q(t)|^{2}+\left[\epsilon_{-}-(1-p)\right]^{2}}}|11\rangle
\end{aligned}
$$


By the use of Eq.(1), the geometric phase becomes,

$$
\phi_{G}=\left(\Omega_{1}+\Omega_{2}\right) \int_{0}^{\tau} d t \frac{p(1-p)|Q(t)|^{2}}{p(1-p)|Q(t)|^{2}+\left[\epsilon_{+}-(1-p)\right]^{2}},
$$

and it is represented in Fig. (8) for an environment of $N=100$ spins. Therein, we can see that the deviation from the unitary geometric phase is less than in the ohmic bosonic environment seen before, but greater than in the supraohmic case. Then, if we consider an experiment of a bipartite system coupled to a spin and photonic environment, we can note that decoherence comes mainly from the spin environment as state in [2] (assuming "sizecomparable" environments).

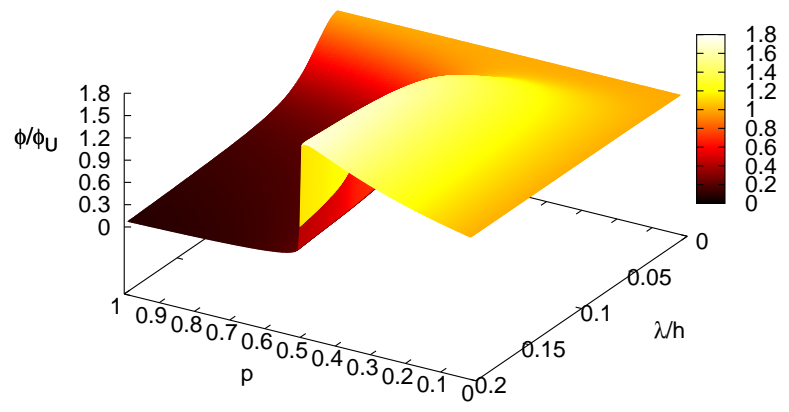

FIG. 8: (colors online) Geometric phase for a bipartite system coupled to a spin environment comprised of $N=100$ spins.

As we have shown for the bosonic environment, the correction to the geometric phase is zero for an initial MES of the composite system, getting a total phase of $\pi$.

It is easy to note that we can reobtain the unitary geometric phase if the bipartite system is isolated. In that case, $\varepsilon_{i}=0=\lambda_{i}$ in $Q(t)$ and the decoherence factor $Q(t)=1$. Similarly, we can check that $\epsilon_{+}=1$ and $\epsilon_{-}=0$. Then the geometric phase becomes

$$
\phi_{G}=\left(\Omega_{1}+\Omega_{2}\right) \int_{0}^{\tau} d t(1-p)=\phi_{G}^{U}=2 \pi(1-p),
$$

assuming $\tau=2 \pi /\left(\Omega_{1}+\Omega_{2}\right)$.

We can perform a series expansion in powers of the coupling with the environment. For that, we shall assume that the couplings between each spin and the environment are equal, i.e. $\varepsilon_{i}=\lambda_{i}$. In order to achieve an analytical result, we shall assume all the bath spins to have the same coupling constant and frequency. Then, the decoherence factor becomes $Q(t)=\prod_{i=1}^{N} q_{i}(t)=q(t)^{N}$ and we can forget about the product function. Besides, we shall perform a perturbative expansion in powers of the dimensionless coupling constant $\lambda / h$. In such a case,

$$
\begin{aligned}
\phi_{G} & \approx 2 \pi(1-p) \\
& +\left(\frac{\lambda}{h}\right)^{2} 16 N p\left(1-3 p+2 p^{2}\right)\left[4 \pi-\frac{\Omega}{h} \sin \left(4 \pi \frac{h}{\Omega}\right)\right],
\end{aligned}
$$

where $\Omega=\Omega_{1}+\Omega_{2}$.

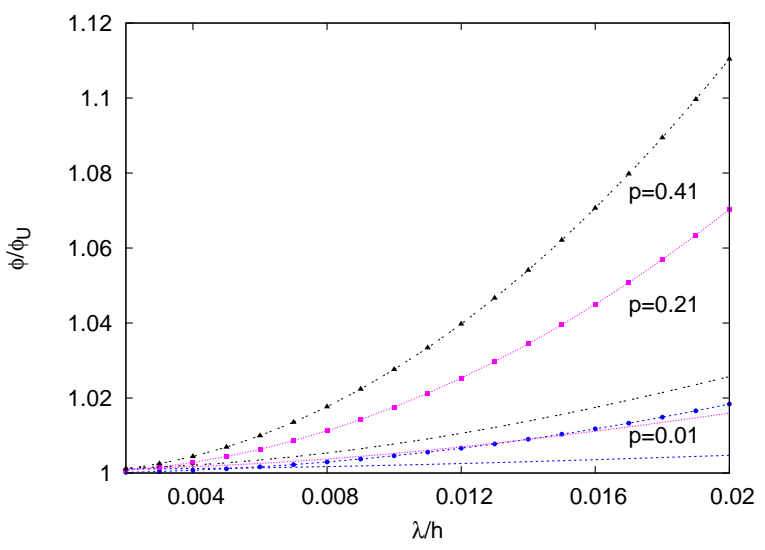

FIG. 9: (colors online) Deviation from the unitary geometric phase for the bipartite system coupled to an environment comprised of $\mathrm{N}=100$ spins as a function of the entanglement of the initial state $\mathrm{p}$ and the coupling constant $\lambda / h$. Exact results are plotted with lines while perturbative ones with lines and dots. Equal colors indicate equal values of $\mathrm{p}$.

In Fig 9 we can see that the series expansion of the geometric phase results a good approximation of the latter for mostly all values of $p$. It is more stressed in the case of small $p N$ since the correction to the phase is quadratic in $\lambda / h$.

\section{Linear Entropy and Concurrence}

As in the other examples analysed for the bosonic environment, we can study the behaviour of the concurrence during the dynamics of the bipartite system. In this case, if we compute the concurrence as explained above, we obtain

$$
\mathcal{C}_{A}=\sqrt{p(1-p)(Q(t)+1)^{2}}-\sqrt{p(1-p)(Q(t)-1)^{2}} .
$$

In Fig 10 and 11 we plot the concurrence and linear entropy as a function of the time for different values of $p$ and the coupling constant $\lambda=\varepsilon$, for an environment comprised of $N=10$ and $N=100$ spins. In both Figures, we can see a peculiar behavior for some of these values. As expected, since we are dealing with a finite environment, information can be in principle recoverable since $\mathrm{Q}(\mathrm{t})$ is at worst quasiperiodic (both proportional 


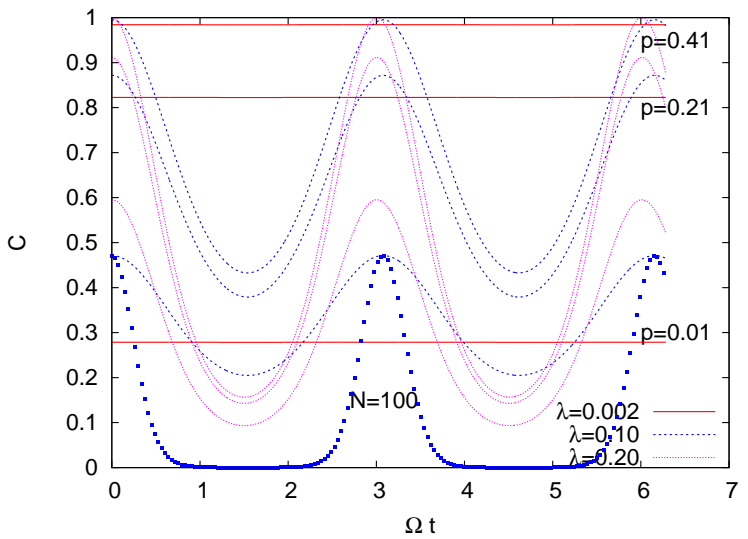

FIG. 10: (colors online) Concurrence as a function of time for different values of the coupling constant $\lambda$ and different values of the initial state $p$, when the bipartite system is coupled to an environment comprised of $N=10$ spins. The dotted line represents the concurrence for $p=0.01, \lambda=0.1$ and $N=100$.

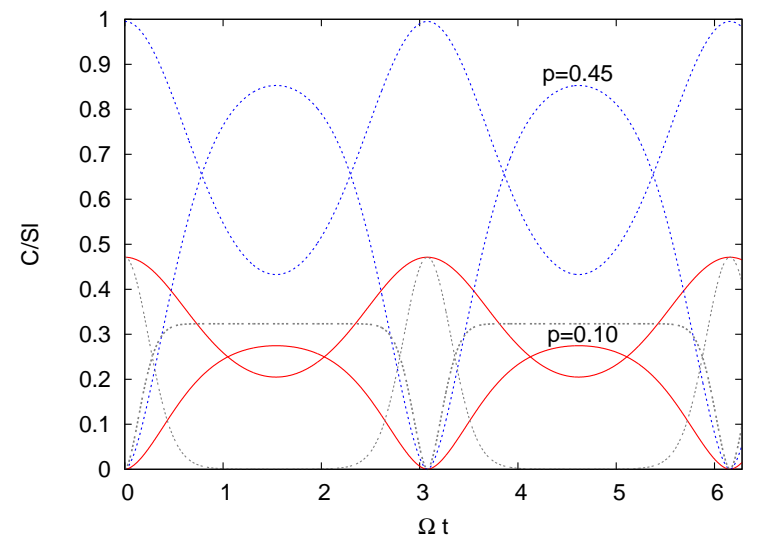

FIG. 11: (colors online) Concurrence and Linear Entropy as a function of time for $\lambda=0.1$ and different values of the initial state $p$, when the bipartite system is coupled to an environment comprissed of $N=10$ spins in the case of $p=$ 0.45 . For $p=0.1$, we present both: an environemt comprised of $N=10$ spins (red solid line) and $N=100$ spins (grey dotted line). to the decoherence factor $\mathrm{Q}(\mathrm{t}))$. As it is well known for this type of environment, the effectiveness of the decoherence mechanism is determined by the dimension of the environment. That is why we observe the periodic behaviour in the analyzed quantities. On the other size, when the coupling with the environment is very small, its presence is not of great importance in the dynamics of the bipartite, and the concurrence is then constant. However, as we increase the coupling constant $\lambda$, we find that the concurrence oscillates due to the oscillating function present in the decoherence factor. In Fig 10 we have plotted the concurrence for different values of $p$ (degree of entanglement of the initial state) with the same color for equal values of the coupling constant $\lambda$. All values considered with lines are for an environment of $N=10$ spins, while the dots represent the concurrence for $p=0.01$ and $\lambda=0.1$ of an environment of $N=100$ spins. We can observe that the effect is stronger when the environment is bigger. Just for the sake of completeness, we plot in Fig 11 the concurrence and linear entropy for two extreme values of the degree of entanglement $p$ for a coupling constant $\lambda=0.1$. In the case of $p=0.1$ we can again observe the difference in both quantities when the size of the environment is $N=10$ (solid line) and when it is $N=100$ (grey dotted line).

\section{B. Werner state with $|\mu\rangle$}

Herein, we shall write $|\mu\rangle$ in Eq.(8). For this initial state, the reduced density matrix has a much simpler expression,

$$
\rho_{\mathrm{rB}}(t)=\left(\begin{array}{cccc}
\frac{(1-r)}{4} & 0 & 0 & 0 \\
0 & \frac{(1-r)}{4}+r(1-p) & r \sqrt{p(1-p)} e^{-i\left(\Omega_{1}+\Omega_{2}\right) t} P(t) & 0 \\
0 & r \sqrt{p(1-p)} e^{i\left(\Omega_{1}+\Omega_{2}\right) t} P^{*}(t) & \frac{(1-r)}{4}+r p & 0 \\
0 & 0 & 0 & \frac{(1-r)}{4}
\end{array}\right)
$$

where the decoherence factor $P(t)$ is

$$
\begin{aligned}
P(t) & =\prod_{i=1}^{N}\left\{1-\left(\frac{2\left(\varepsilon_{i}-\lambda_{i}\right)^{2}}{h_{i}^{2}+\left(\varepsilon_{i}-\lambda_{i}\right)^{2}}\right)\right. \\
& \left.\times \sin ^{2}\left(t \sqrt{h_{i}^{2}+\left(\varepsilon_{i}-\lambda_{i}\right)^{2}}\right)\right\} .
\end{aligned}
$$

has been defined in the Appendix B.

In order to compute the geometric phase, we need to know the eigenvalues of the reduced density matrix. 
These are (for $r=1$ )

$$
\begin{aligned}
\epsilon_{1,2} & =0 \\
\epsilon_{ \pm} & =\frac{1}{2}\left[1 \pm \sqrt{1+4 p(1-p)\left(|P(t)|^{2}-1\right)}\right]
\end{aligned}
$$

and the corresponding eigenvectors that again can be easily computed.

By the use of Eq.(1), the geometric phase becomes,

$$
\phi_{G}=\Omega \int_{0}^{\tau} d t \frac{p(1-p)|P(t)|^{2}}{p(1-p)|P(t)|^{2}+\left[\epsilon_{+}-(1-p)\right]^{2}},
$$

where $\Omega=\left(\Omega_{1}-\Omega_{2}\right)$. It is easy to note that we can reobtain the unitary geometric phase if the bipartite system is isolated or if it is in a MES with $p=1 / 2$. In isolated from the environment case, $\varepsilon_{i}=0=\lambda_{i}$ in $P(t)$ and the decoherence factor $P(t)=1$. Similarly, we can check that $\epsilon_{+}=1$ and $\epsilon_{-}=0$. Then the geometric phase becomes

$$
\phi_{G}=\Omega \int_{0}^{\tau} d t(1-p)=\phi_{G}^{U}=2 \pi(1-p),
$$

assuming $\tau=2 \pi / \Omega$, once again.

The concurrence for this case can be also calculated as

$$
\mathcal{C}_{B}=\sqrt{p(1-p)(P(t)+1)^{2}}-\sqrt{p(1-p)(P(t)-1)^{2}} .
$$

As it is easy to note, this case is similar to the one presented before but changing the decoherence factor $Q(t) \rightarrow P(t)$. However this fact is not trivial. If we consider that the coupling between each spin of the system and the external reservoir is equal, i.e. $\varepsilon_{i}=\lambda_{i}$, the $P(t)=1$ and the subspace generated by the states $|01\rangle$ and $|10\rangle$ becomes a decoherence free subspace, reobtaining the unitary geometric phase and a concurrence $\mathcal{C}_{B}=2 \sqrt{p(1-p)}$.

\section{CONCLUSIONS}

The geometric phase of entangled states is an issue worth of attention. It could be a potential application in holonomic quantum computation since the study of entangled spin systems effectively allows us to contemplate the design of a solid state quantum computer. However, decoherence is the main obstacle to overcome. All realistic quantum systems are coupled to their surroundings to a greater or lesser extent.

We have thoroughly studied the geometric phase for a bipartite system, i.e. two coupled qubits, also coupled to an external environment. We have considered both cases: a bosonic environment at zero temperature (whether ohmic or supraohmic) and a spin environment. In all cases, we have chosen a general initial state and computed the evolution of the composite system in terms of the reduced density matrix. We have further estimated the decoherence factors for all cases in order to have a full insight into the decoherence process induced by the environment on the system's dynamics. We have seen that there is a hierarchy among the environments. That means, when considering real systems, such as single molecule magnets (for example $\mathrm{Fe}_{8}$ molecule), decoherence induced by the nonlocalized modes of a supraohmic environment is less than that induced by the localized dicrete modes of a spin environment.

By the use of a kinematic approach, we have computed the geometric phase $\phi_{G}=\phi_{G}^{U}+\delta \phi_{G}$ for different choices of the initial state of the bipartite system. In some cases, we have also performed a perturbative expansion of the geometric phase in order to complete the analysis. In all cases, we have found the same geometric dependence upon the initial angle $\theta_{0}$ for the correction of the geometric phase that has been found by some authors by the use of different approaches [13].

As entanglement is considered to be one of the key resources in quantum information science, we have also studied this property of the composite system. In particular, we have checked that the correction to the geometric phase is $\delta \phi_{G}=0$ for the case of maximally entangled initial states of the bipartite systems. This is the case for all the cases considered, no matter the kind of environment would be present so far.

We have also computed the concurrence and linear entropy of each initial state. We find a steady relation between the geometric phase and the concurrence only for a Bell state in the isolated situation. In all other cases, not only the geometric phase but also the concurrence are modified but the presence of the environment.

In all cases it is posible to compute the geometric phase in not only one cyclic but many, i.e. $\tau=n 2 \pi / \Omega$, with $n$ integer. In that case, the correction to the unitary geometric phase would be proportional to $n$, the winding number [12, 13]. This proportionality of $\delta \phi_{G}$ to $n$ might imply that the geometric phase of the composite system still has some of its geometric character. However, this correction is also a function of the environments spectrum and then the total geometric phase is not a simple geometric quantity.

\section{Appendix A: Derivation of the reduced density matrix for a bosonic environment}

Herein, we shall derive the reduced density matrix for a two spin $1 / 2$ particles coupled to a bosonic environment through coupling constants $\lambda_{n}$ for the spin 1 and $g_{n}$ for spin 2 (being $\lambda_{n}$ not necessarily equal to $g_{n}$ ).

In order to know the dynamics of the bipartite system, we shall find the reduced density matrix for the composite system, as has been done in [25] for one particle spin-boson model in the case of weak coupling. We must note that in this case the interaction is $\tilde{\mathrm{V}}\left(t_{1}\right)=$ $\sigma_{z}^{1} \sum_{n} \lambda_{n} q_{n}\left(t_{1}\right)+\sigma_{z}^{2} \sum_{n} g_{n} q_{n}\left(t_{1}\right)$. Similarly to the single spin-boson model, it is straightforward to compute 
the time derivative of the reduced density matrix as (we have set $\hbar=1$ from here up to what follows):

$$
\dot{\tilde{\rho}}_{\mathrm{r}}=-\int_{0}^{t} d t_{1} \operatorname{Tr}_{\mathrm{B}}\left[\tilde{\mathrm{V}}\left(t_{1}\right),\left[\tilde{\mathrm{V}}\left(t_{1}\right), \tilde{\rho}_{r} \otimes \tilde{\rho}_{B}(0)\right]\right] .
$$

The tilde indicates that we are working in the Interaction Picture.

After doing some algebra, the master equation for the reduced density matrix can be written as

$$
\begin{aligned}
\dot{\rho}_{\mathrm{r}}(t) & =-i\left[H_{s}, \rho_{r}\right] \\
& -\int_{0}^{t} d t_{1}\left\{\nu _ { 1 } ( t _ { 1 } ) \left[\sigma_{z}^{1},\left[\sigma_{z}^{1}, \rho_{r}\right]+\nu_{2}\left(t_{1}\right)\left[\sigma_{z}^{2},\left[\sigma_{z}^{2}, \rho_{r}\right]\right]\right.\right. \\
& \left.+\nu_{12}\left(t_{1}\right)\left(\left[\sigma_{z}^{1},\left[\sigma_{z}^{2}, \rho_{r}\right]\right]+\left[\sigma_{z}^{2},\left[\sigma_{z}^{1}, \rho_{r}\right]\right]\right)\right\} \\
& +i \int_{0}^{t} d t_{1} \eta_{12}\left(t_{1}\right)\left(\left[\sigma_{z}^{1},\left\{\sigma_{z}^{2}, \rho_{r}\right\}\right]\right. \\
& \left.+\left[\sigma_{z}^{2},\left\{\sigma_{z}^{1}, \rho_{r}\right\}\right]\right),
\end{aligned}
$$

where we have already taken the continuum limit and defined the noise and dissipation kernels as:

$$
\begin{aligned}
& \nu_{i}(t)=\int_{0}^{\infty} d \omega J_{i}(\omega) \cos (\omega t) \operatorname{coth}\left(\frac{\beta \omega}{2}\right), \\
& \eta_{i}(t)=\int_{0}^{\infty} d \omega J_{i}(\omega) \sin (\omega t),
\end{aligned}
$$

with $i=1,2$ or $i=12$. $J_{i}(\omega)$ is the spectral density of the environment associated to each spin of the system, and $\nu_{i}(t)$ the corresponding noise kernel while $\eta_{i}(t)$ is the dissipation kernel associated to $J_{i}(\omega)$. One assumption we shall make is that $J(\omega)$ is a reasonably smooth function of $\omega$, and that is of the form $\omega^{n}$ up to some frequency $\Lambda$ that may be large compared to $\Omega_{1}$ and $\Omega_{2}$. The spectral density function can be written as $J_{i}(\omega)=\gamma_{0 i} / 4 \omega^{n} \Lambda^{n-1} e^{-\omega / \Lambda}[\underline{3}]$. Notice that at this stage, the coupling constants of the model $\lambda_{n}$ and $g_{n}$ have been absorbed in the continuous limit and that information is now contained in the dimensionless dissipative constants $\gamma_{01}, \gamma_{02}$, and $\gamma_{012}$ respectively, defined in the spectral density of the bath.
In Eq. (A1) we note the first difference with the dephasing single spin-boson [10, 25]. In the case of only one spin coupled to an external environment, there is no dissipation on the main system induced by the environment (it is just a pure dephasing model). However, in the bipartite system we see that dissipation appears dispite of the similar coupling between the system and the environment.

In this context, we can define the decoherence factors $\Gamma_{i}(t)$ as

with

$$
\Gamma_{i}(t)=e^{-4 \int_{0}^{t} d t_{1} F_{i}\left(t_{1}\right)},
$$

$$
F_{i}(t)=\int_{0}^{t} d t^{\prime} \nu_{i}\left(t^{\prime}\right)
$$

and the dissipation induced by the environment as

$$
G_{12}(t)=\int_{0}^{t} d t^{\prime} \eta_{12}\left(t^{\prime}\right)
$$

\section{Appendix B: Derivation of the reduced density matrix for an environment comprised of spins}

Herein, we shall derive the reduced density matrix for a bipartite system coupled to an environment comprised of $N$ spins. The system's, environment's and interaction hamiltonians have been considered in the main text, Sec

Let's take an initial separable state of the complete system, i.e. $|\Psi(0)\rangle=|\Phi(0)\rangle \otimes|\chi(0)\rangle$, where $|\Phi(0)\rangle$ is the initial state of the bipartite system while $|\chi(0)\rangle$ is for the spin environment. We can note that $\left[H_{S}, H_{I}\right]=0$, so we can write the evolution of the complete state as

$$
\begin{aligned}
|\Psi(t)\rangle & =e^{-i\left(H_{S}+H_{I}+H_{B}\right) t}|\Psi(0)\rangle \\
& =e^{-i\left(H_{S}+H_{I}\right) t}|\Phi(0)\rangle \otimes e^{-i\left(H_{B}\right) t}|\chi(0)\rangle .
\end{aligned}
$$

After cumbersome calculations (that we do not consider necessary to be presented here), we have obtained the following reduced density matrix for a general initial state $|\Phi(0)\rangle=\alpha|00\rangle+\beta|01\rangle+\zeta|10\rangle+\delta|11\rangle$ of the bipartite system

$$
\rho_{\mathrm{r}}(t)=\left(\begin{array}{cccc}
|\alpha|^{2} & \alpha \beta^{*} e^{-i\left(\Omega_{2}+2 \gamma\right) t} M(t) & \alpha \zeta^{*} e^{-i\left(\Omega_{1}+2 \gamma\right) t} N(t) & \alpha \delta^{*} e^{-i\left(\Omega_{2}+\Omega_{1}\right) t} Q(t) \\
\alpha^{*} \beta e^{i\left(\Omega_{2}+2 \gamma\right) t} M^{*}(t) & |\beta|^{2} & \beta \zeta^{*} e^{-i\left(\Omega_{1}-\Omega_{2}\right) t} P(t) & \beta \delta^{*} e^{-i\left(\Omega_{1}-2 \gamma\right) t} R(t) \\
\alpha^{*} \zeta e^{i\left(\Omega_{1}+2 \gamma\right) t} N^{*}(t) & \zeta \beta^{*} e^{i\left(\Omega_{1}-\Omega_{2}\right) t} P^{*}(t) & |\zeta|^{2} & \zeta \delta^{*} e^{-i\left(\Omega_{2}-2 \gamma\right) t} S(t) \\
\alpha^{*} \delta e^{i\left(\Omega_{2}+\Omega_{1}\right) t} Q^{*}(t) & \delta \beta^{*} e^{i\left(\Omega_{1}-2 \gamma\right) t} R^{*}(t) & \delta \zeta^{*} e^{i\left(\Omega_{2}-2 \gamma\right) t} S^{*}(t) & |\delta|^{2}
\end{array}\right)
$$


decoherence factors $M(t), \quad N(t), \quad P(t), \quad Q(t), \quad R(t)$, and $S(t)$. These factors have several expressions. $M(t)=\operatorname{Tr}_{B}\left[e^{-i\left(H_{B}+V_{s}\right) t} \rho_{B}(0) e^{i\left(H_{B}+V_{r}\right) t}\right]$, with $V_{s}=\left(\varepsilon_{i}+\lambda_{i}\right)$ and $V_{r}=\left(\varepsilon_{i}-\lambda_{i}\right)$. Similarly, $N(t)=\operatorname{Tr}_{B}\left[e^{-i\left(H_{B}+V_{s}\right) t} \rho_{B}(0) e^{i\left(H_{B}-V_{r}\right) t}\right]$, $S(t)=\operatorname{Tr}_{B}\left[e^{-i\left(H_{B}+V_{r}\right) t} \rho_{B}(0) e^{i\left(H_{B}-V_{s}\right) t}\right]$, $R(t)=\operatorname{Tr}_{B}\left[e^{-i\left(H_{B}+V_{r}\right) t} \rho_{B}(0) e^{i\left(H_{B}-V_{s}\right) t}\right]$, $Q(t)=\operatorname{Tr}_{B}\left[e^{-i\left(H_{B}+V_{s}\right) t} \rho_{B}(0) e^{i\left(H_{B}-V_{s}\right) t}\right]$ and $P(t)=\operatorname{Tr}_{B}\left[e^{-i\left(H_{B}+V_{r}\right) t} \rho_{B}(0) e^{i\left(H_{B}-V_{r}\right) t}\right]$. In this paper, we shall only be interested in the last two coefficients. Since we can choose a pure state for the initial state of the bath as $|\chi(0)\rangle=\prod_{i=1}^{N}\left(\alpha_{i}\left|0_{i}\right\rangle+\beta_{i}\left|1_{i}\right\rangle\right)$, then $\rho_{B}(0)=|\chi(0)\rangle\langle\chi(0)|$. In order to have closed expressions for these coefficients, we have finally assumed $\left|\alpha_{i}\right|=\left|\beta_{i}\right|$ and obtained the following decoherence factors

$$
\begin{aligned}
Q(t) & =\prod_{i=1}^{N}\left\{1-\left(\frac{2\left(\varepsilon_{i}+\lambda_{i}\right)^{2}}{h_{i}^{2}+\left(\varepsilon_{i}+\lambda_{i}\right)^{2}}\right)\right. \\
& \left.\times \sin ^{2}\left(t \sqrt{h_{i}^{2}+\left(\varepsilon_{i}+\lambda_{i}\right)^{2}}\right)\right\} \\
P(t) & =\prod_{i=1}^{N}\left\{1-\left(\frac{2\left(\varepsilon_{i}-\lambda_{i}\right)^{2}}{h_{i}^{2}+\left(\varepsilon_{i}-\lambda_{i}\right)^{2}}\right)\right. \\
& \left.\times \sin ^{2}\left(t \sqrt{h_{i}^{2}+\left(\varepsilon_{i}-\lambda_{i}\right)^{2}}\right)\right\} .
\end{aligned}
$$

We can find some common features between these expressions and preexisting Literature. For example, we can note that the factor $P(t)$ that affects the states $|01\rangle$ and $|10\rangle$ is similar to that found in [12] for a two-level system coupled to an external environment. In that same case, if we do not consider the self interaction of the bath, i.e. $h_{i}=0$, then we get $P(t)=\prod_{i=1}^{N} \cos \left[2\left(\varepsilon_{i}+\lambda_{i}\right) t\right]$ as found in [4, 11]. It is also possible to check that $P(0)=1$. As it was done in [4], we can evaluate the mean value of the decoherence factor. As in that case, $\langle P(t)\rangle_{T \rightarrow \infty} \rightarrow 0$. If we estimate the average dispersion as $\sigma^{2}=\left\langle P(t)^{2}\right\rangle-\langle P(t)\rangle^{2}=\sum_{i=1}^{N} p_{i}$, with $p_{i}=\left(1-\frac{\left(\varepsilon_{i}+\lambda_{i}\right)^{2}}{4\left(h_{i}^{2}+\left(\varepsilon_{i}+\lambda_{i}\right)^{2}\right)}\right)$. Under the assumption that all $p_{i}$ are approximately equal, the average fluctuations from zero are

$$
\sigma \sim \frac{1}{\sqrt{N}}
$$

Therefore, large environments can effectively induce decoherence on the central spin system. We can do the same with all the decoherence factors but in the following we shall only be interested in factors $P(t)$ and $Q(t)$.

Acknowledgments. This work was supported by UBA, CONICET, and ANPCyT, Argentina.
[1] G. Vidal, Phys. Rev. Lett. 91, 147902 (2003); Phys. Rev. Lett. 93, 040502, (2004).

[2] Philip C.E. Stamp and Alejandro Gaita-Ariño, J. Mat. Chem. 19, 1718 (2009).

[3] A.J. Leggett, S. Chakravarty, A.T. Dorsey, M.P.A. Fisher, A. Garg, and W. Zwerger, Rev. Mod. Phys. 59, 1 (1987).

[4] W.H. Zurek, Phys. Rev. D 26, 1862 (1982).

[5] Maximilian Schlosshauer, Decoherence and the Quantum-To-Classical Transition, Springer (2007).

[6] S. Pancharatnam, Proc. Indian Acad. Sci. A 44, 247 (1956).

[7] M.V. Berry, Proc. R. Soc. Lond. A 392, 45 (1984).

[8] Y. Aharonov, J. Anandan, Phys. Rev. Lett. 58, 1593 (1988); J. Anandan, Y. Aharonov, Phys. Rev. D 38, 1863 (1988).

[9] D. M. Tong, E. Sjoqvist, L. C. Kwek, and C. H. Oh, Phys. Rev. Lett. 93, 080405 (2004); see also Phys. Rev. Lett. 95, 249902 (2005).

[10] F.C. Lombardo and P.I. Villar, Phys. Rev. A 74, 042311 (2006).

[11] F.C. Lombardo and P.I. Villar, Int. J. of Quantum Information 6, 707713 (2008).

[12] Paula I. Villar, Phys. Lett. A 373, 206 (2009).

[13] R.S. Whitney and Y. Gefen, Phys. Rev. Lett. 90, 190402, (2003); R.S. Whitney, Y. Makhlin, A. Shnirman, and Y. Gefen, Phys. Rev. Lett. 94, 070407 (2005).

[14] A. Carollo, I. Fuentes-Guridi, M. Franca Santos, and V. Vedral, Phys. Rev. Lett. 90, 160402 (2003); Phys. Rev.
Lett. 92, 020402 (2004).

[15] G. De Chiara, A. Lozinski, G. M. Palma, Eur. Phys. J. D 41, 179-183 (2007); G. De Chiara, G. M. Palma, Phys. Rev. Lett. 91, 090404 (2003).

[16] S. Benerjee and R. Skikanth, Eur. Phys. J. D 46, 335 (2008).

[17] A.T. Rezakhani and P. Zanardi, Phys. Rev. A 73, 012107 (2006).

[18] Pérola Milman and Rémy Mosseri, Phys. Rev. Lett. 90, 230403 (2003); Pérola Milman, Phys. Rev. A 73, 062118 (2006).

[19] J. Batle, M. Casas, A. Plastino, A. R. Plastino, Phys. Lett. A 343, 12 (2005).

[20] H. T. Cui, L.C. Wang, and X.X.Yi, Eur. Phys. J. D 41, 385 (2007).

[21] E. Sjoqvist, Arun K. Pati, Artur Ekert, Jeeva S. Anandan, Marie Ericsson, Daniel K. L. Oi, and Vlatko Vedral, Phys. Rev . Lett. 85, 2845 (2000).

[22] F.C. Lombardo and P.I. Villar, Phys. Lett. A 371, 190 (2007).

[23] W. K. Wootters, Phys. Rev. Lett. 80, 2245(1998).

[24] B. Basu, Europhys. Lett. 73, 833 (2006).

[25] J. P. Paz and W. H. Zurek, Environment induced superselection and the transition from quantum to classical in Coherent matter waves, Les Houches Session LXXII, edited by R. Kaiser, C. Westbrook and F. David, EDP Sciences, Springer Verlag (Berlin) (2001) 533-614; W.H. Zurek, Rev. Mod. Phys. 75, 715 (2003). 